\title{
A Visual Comparative Study of Multi-Objective Transportation Problem in Several Approaches
}

\author{
M. I. Moussa \\ Computer Science Department, Faculty of Computers \& Informatics, Benha University, Egypt, \\ Email: moussa_6060@yahoo.com
}

\section{ABSTRACT}

The main objective of this study was to present in one applet java program the five approaches for solving the MultiObjective Transportation Problem (MOTP). The program was built using the java programming language, to solve the MOTP and to visualize the solution steps. In addition, the study made a comparisons between the four different approaches used to solve the MOTP. For better understanding, the solution procedure is illustrated with a numerical example.

\section{Keywords}

Multi-objective optimization; Genetic algorithm; java.

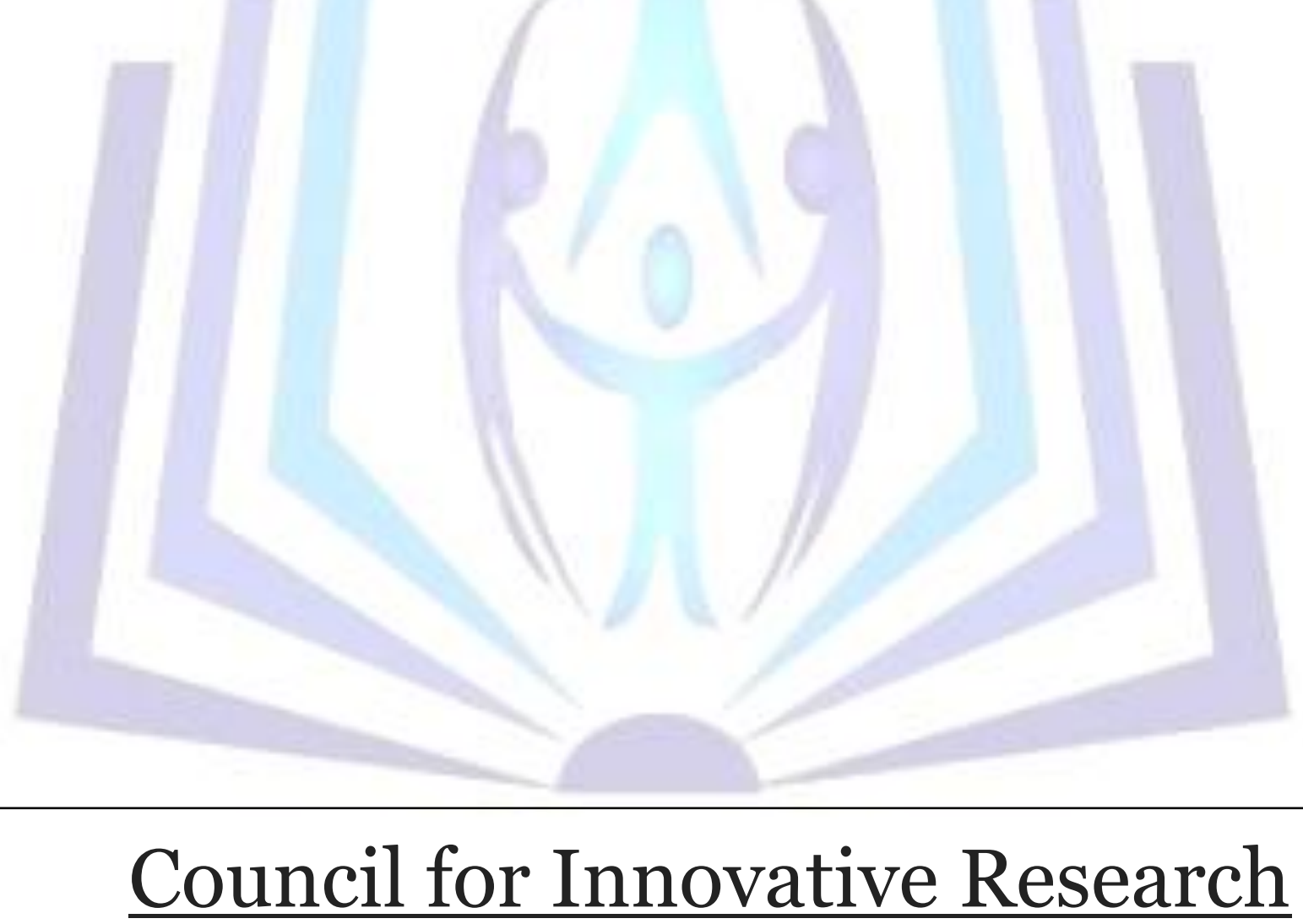

Peer Review Research Publishing System

Journal: International Journal of Management \& Information Technology

Vol. 7, No. 1

editor@cirworld.com

www.cirworld.com, member.cirworld.com 


\section{INTRODUCTION}

The Transportation Problem (TP) is a special type of linear programming problem. It is considered as a minimum cost flow problem, which deals with the transportation of commodities from $\mathrm{m}$ sources to $\mathrm{n}$ destinations. This means to transfer a quantity of products from plants to warehouses such that the transportation cost is minimum. TP usually involves a single objective function. However the transportation problem which involves multiple objective functions is called Multi-Objective Transportation Problem (MOTP).

A number of studies has been available to obtain an optimal solution for balanced transportation problems. That is when the total supply is equal to the total demand then the transportation model is said to be balanced [23]. But in real life, the decision maker faces an unbalanced transportation problem in which the total supply is less than the total demand. This can be solved through compromised solutions. M. Zangiabadi and H. R. Maleki used a special type of nonlinear (hyperbolic and exponential) membership functions to solve MOTP, it gives an optimal compromise solution [17]. Kishore and Jayswal [10] introduced a method, called fuzzy approach, to solve unbalanced transportation problems with budgetary constraints. Charnsethikul Peerayuth and Sverasreni Saeree [3] discussed a method for solving the constrained bottleneck transportation problem under budgetary condition. Lin and Cheng [14] gave a genetic algorithm for solving a transportation network under a budget Constraint. Senapati and Tapan Kumar [22] investigated fuzzy multi-index transportation problems with budgetary restriction. Khanna, Bak hshiand Puri [9] introduced an algorithm for solving transportation flow under budgetary constraints. Tiwari, Dharmar and Rao [24] investigated how the preemptive priority structure can be used in fuzzy goal programming problems. Weighted goal programming for unbalanced single objective transportation problem with budgetary constraint has been discussed by Kishore and Jayswal [11]. Pandian and Natarajan [18] introduced the zero point method for finding an optimal solution to a classical transportation problem. Subsequently, a number of transportation problems have been appeared in the literature $[15,7,6,21,8]$.

The main objectives of this study were to present in one applet java program the four approaches:

- Interactive algorithmsI ( Interactive algorithmsII)[20].

- A fuzzy approach[1,16].

- Interactive a fuzzy goal programming[2,19].

- Pareto-based approach[23].

The study solved the MOTP and made a comparison between the four approaches. The program was built using the java programming language, to solve the MOTP and to visualize the solution steps. The study addressed the following steps: The first was to solve MOTP using the five approaches. The second was to combine the genetic algorithm and the five approaches. The third was to provide a visual solution which compare between the five approaches.

The remainder of this paper is organized as follows. In section (2) some basic concepts and definitions about the MOTP and the genetic algorithm were introduced. In section (3) the study described five methods which have been used to solve the MOTP. Section (4) showed the implementation of the problem and results. Section (5) analysed the results and compared between the the different solutions of MOTP. Section (6) is the conclusion of this research.

\section{PRELIMINARIES}

Given a transportation model with $\mathrm{m}$ sources and $\mathrm{n}$ destinations, the amount of supply available at source $\mathrm{i}$ is ai, the demand required at destination $\mathrm{j}$ is $\mathrm{bj}$, the cost of transporting one unit between source $\mathrm{i}$ and destination $\mathrm{j}$ is cij and xij denotes the quantity transported from source $i$ to destination $j$ so the cost associated with this movement is cost $\times$ quantity $=c_{i j} x_{i j}$. The cost of transporting the commodity from source $\mathrm{i}$ to all destinations is given by

$$
\sum_{j=1}^{n} c_{i j} x_{i j}=c_{i 1} x_{i 1}+c_{i 2} x_{i 2}+c_{i 3} x_{i 3}+\ldots+c_{i n} x_{i n}
$$

Thus, the total cost $F\left(X_{*}\right)$ of transporting the commodity from all the sources to

all the destinations is

$$
F\left(X_{*}\right)=\sum_{i=1}^{m} \sum_{j=1}^{n} c_{i j} x_{i j}
$$

The sources may be factories, warehouses, etc. and they are characterized by available quantities denoted $a_{1}, \ldots, a_{m}$. The destination may be warehouses, sales outlets, etc. and they are characterized by available 
quantities denoted $b_{1}, \ldots, b_{n}$. The transportation cost between a given source $i$ to a given destination $j$ pair is the penalty $c_{i j}$. The unknown quantity to be transported between the source-destination pair $(i, j)$ denoted $x_{i j}$. In the simplest case, the unit transportation cost is constant. The transportation problem is to find the optimal distribution plan for shipments from sources to destinations that minimizes the total transportation cost, in the same time, it seeks to find an optimal distribution plan for a single commodity. The mathematical form of MOTP can be stated as follows:

$$
P_{1} \min \quad F^{k}\left(X_{*}\right)=\sum_{i=1}^{m} \sum_{j=1}^{n} c_{i j} x_{i j}
$$

Subject to

$$
\begin{gathered}
\sum_{j=1}^{n} x_{i j}=a_{i} \quad i=1,2, \ldots, m \\
\sum_{i=1}^{m} x_{i j}=b_{j} \quad j=1,2, \ldots, n \\
x_{i j} \geq 0, \quad i=1,2, \ldots, m, \quad j=1,2, \ldots, n, \quad k=1,2, \ldots, K
\end{gathered}
$$

Where $F^{k}\left(X_{*}\right)$ is the multiple objective functions, it is a vector of $k$ objective functions and $X_{*}$ is a feasible vector solution of $P_{1}$. The above MOTP form is knewn as the canonical form, by changing the pair of equality in(4) and (5) to a pair of inequality, it results the equivalent problem which is in standard form. Every minimization problem can be appeared as a maximization problem and vice versa.

Genetic algorithm has shown a great potential to work out several real-world problems in the point of optimization. One of these problems is MOTP. That algorithmic model begins with the creation of a set of solutions which are referred to as a population of individuals. Each individual in a population consists of a set of parameter values which completely describe a solution. A solution is encoded in a string called a chromosome, which consists of genes that can take a number of values. Initially, the collection of solutions (population) is generated randomly and at each iteration a new generation of solutions is formed by applying genetic operators (crossover, mutation, selection). Each solution is evaluated using an objective function called a fitness function and this process is repeated until some form of convergence in fitness is achieved. The goal of the optimization process is to minimize or maximize the fitness.

Dominated Solution: A solution $X_{(1)}$ is said to dominate the other solution $X_{(2)}$, if both conditions 1 and 2 are true: The solution $X_{(1)}$ is no worse than $X_{(2)}$ in all objectives, or no value of $F^{j}\left(X_{(1)}\right)$ is greater than any value of $F^{j}\left(X_{(2)}\right)$ for all $j=1,2 \ldots M$.

The solution $X_{(1)}$ is strictly better than $X_{(2)}$ in at least one objective, or $F^{j}\left(X_{(1)}\right)<F j\left(X_{(2)}\right)$ for at least one $j \in\{1,2 \ldots M\}$.

Nondominated Set [20 ]: Among a set of solutions $S$ of $P_{1}$, the non-dominated set of solutions $S$ ' are those that are not dominated by any member of the set $S$.

deal Solution [1]: For the MOTP, the ideal solution is the point in the outcome space which represents the vector $\left(F^{1}\left(X_{o(i)}\right), F^{2}\left(X_{o(i)}\right), \ldots, F^{k}\left(X_{o(i)}\right)\right)$ where $F^{i}\left(X_{o(i)}\right)$ denotes an optimal value for the i-th function $F^{i}\left(X_{o(i)}\right)$ and $X_{o(i)}=\left(x_{o(i)}^{1}, x_{o(i)}^{2}, \ldots, x_{o(i)}^{n}\right)$ is the vector of variables which optimizes $F^{i}\left(X_{o(i)}\right)$

Compromise Solution [7]: A feasible vector $X_{*} \in S$ is called a compromise solution of $P_{1}$ if and only if $X_{*}$ have the minimum deviation from the ideal point than any other point in $S$. Thus, the compromise solution is the closest solution to the ideal one that maximizes the underlying utility function of the decision maker. If the compromise solution satisfies the decision maker's preferences, then the solution is called the preferred compromise solution. 
Distance Functions [1]: Assuming $\sum_{k=1}^{K} \lambda_{k}=1$, where $\lambda=\left(\lambda_{1}, \lambda_{2} \ldots \lambda_{\mathrm{K}}\right)$ is the vector of objectives aspiration levels, the family of distance functions $D_{p}(\lambda, k)$ to measure the degree of closeness of the compromise solution to the ideal solution is stated as follows:

$$
D_{p}(\lambda, k)=\left[\sum_{k=1}^{K} \lambda_{k} p\left(1-d_{k}\right)\right]
$$

Where the power $\mathrm{p}$ represents a distance parameter $1 \leq \mathrm{p} \leq \infty, d_{k}$ is the degree of closeness of the preferred compromise solution vector $X_{*}$ to the optimal solution vector with respect to the k-th objective function, and in minimum problem $d_{k}$ equals:

$$
d_{k}=\frac{\text { The optimal solution of } F^{k}}{\text { The preferred compromise solution of } F^{k}}
$$

The distance functions with $p=1,2$, and $\infty$ are

$$
D_{1}(\lambda, k)=1-\sum_{k=1}^{K} \lambda_{k} d_{k}, \quad D_{2}(\lambda, k)=\left[\sum_{k=1}^{K} \lambda_{k}^{2}\left(1-d_{k}\right)^{2}\right]^{1 / 2} \text { and } D_{-\mathrm{inf}}(\lambda, k)=\max _{k}\left\{\lambda_{k}\left(1-d_{k}\right)\right\}
$$

\section{EXPERIMENTAL, RESULTS AND DISCUSSIONS}

The four solution's approaches for solving MOTP and the genetic algorithm in java language were implemented to obtain the optimal solution for each approach. Also, practical comparisons between the four approaches were presented. Figure 1( A) gives the user interface of the JAVA-APPLET program. The input to the frame shows transport products from three sources to four destinations center. The user started to input the general data of the problem that determines the size of the problem, the number of the multi-objective functions of the optimization problem and population size (the population size is the number of chromosomes in the population). The data that describe the details of the problem are entered through two buttons on this frame; data from frame and data from frame and file. "Minimum or Maximum" buttons determine the type of the problem. This frame is revealing in the four solution approaches for the MOTP.

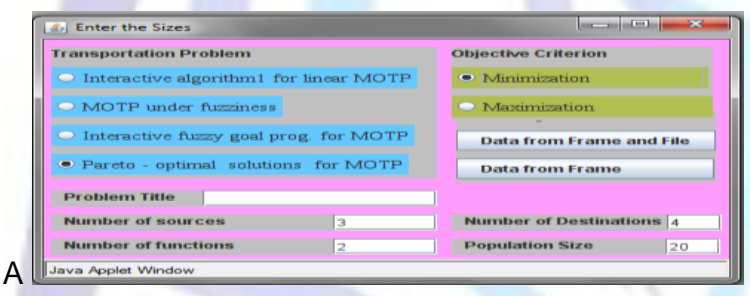

Fig 1: (A) The general data of MOTP

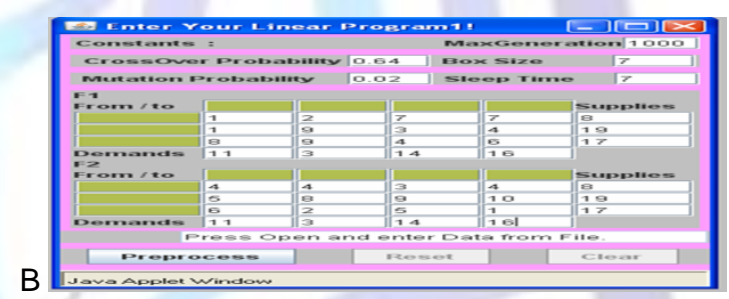

(B) Example 1 in matrix notation

Example1: Consider MOTP with the following characteristics.

Supplies: $a_{1}=8, a_{2}=19, a_{3}=17$ Demands: $b_{1}=11, b_{2}=3, b_{3}=14, b_{4}=16$

Penalties :

$$
C^{1}=\left[\begin{array}{llll}
1 & 2 & 7 & 7 \\
1 & 9 & 3 & 4 \\
8 & 9 & 4 & 6
\end{array}\right] \quad C^{2}=\left[\begin{array}{llll}
4 & 4 & 3 & 4 \\
5 & 8 & 9 & 10 \\
6 & 2 & 5 & 1
\end{array}\right]
$$

The optimal solution for each function and the corresponding point in the outcome space is: $F^{1}\left(X_{1}\right)=143, F^{2}\left(X_{2}\right)=167$, $X_{1}=(5,3,0,0,6,0,0,13,0,0,14,3)$, and $X_{2}=(0,0,8,0,11,2,6,0,0,1,0,16)$.

Unknown variable $x_{i}$ in the feasible solution of the problem represents a gene in the appropriate design of chromosomes (Figure 2). Each chromosome consists of a sequence of $m$ sub-chromosomes ( $m$ is the number of supplies). Each sub-chromosome consists of $n$ genes ( $n$ is the number of demands). In Example1, there are three supplies $(m=3)$ and four demands $(n=4)$, so the feasible solution is $\left(x_{1}, x_{2}, \ldots, x_{12}\right)$. 
Table 1 Numerical values of F1; F2, D1, D2 and $D_{- \text {inf }}$ in example 1 according to the four approaches

\begin{tabular}{|l|l|l|l|l|l|}
\hline & $\begin{array}{l}\text { Interactive(I) } \\
\text { approach }\end{array}$ & Fuzzy approach & $\begin{array}{l}\text { Interactive Fuzzy goal } \\
\text { programming approach }\end{array}$ & $\begin{array}{l}\text { Pareto-based } \\
\text { approach }\end{array}$ & $\begin{array}{l}\text { Optimal } \\
\text { solution }\end{array}$ \\
\hline$F_{1}$ & 176 & 160 & 160 & 190 & 143 \\
\hline$F_{2}$ & 175 & 195 & 195 & 164 & 167 \\
\hline$D_{1}$ & 0.116607 & 0.12492 & 0.12492 & 0.124551 & --- \\
\hline$D_{2}$ & 0.096496 & 0.089313 & 0.089313 & 0.088105 & --- \\
\hline$D_{- \text {inf }}$ & 0.09375 & 0.071795 & 0.071795 & 0.064024 & --- \\
\hline
\end{tabular}

The structure of the chromosome corresponding to this solution concatenated the twelve variable $x_{i}$ in a binary numbers; 0101, 11, 0001, 1011, 0101, 11, 0111, 01111, 0101, 01, 0110, and 01011. These give one chromosome as; C:01011100011011010111011101111010101011001011.

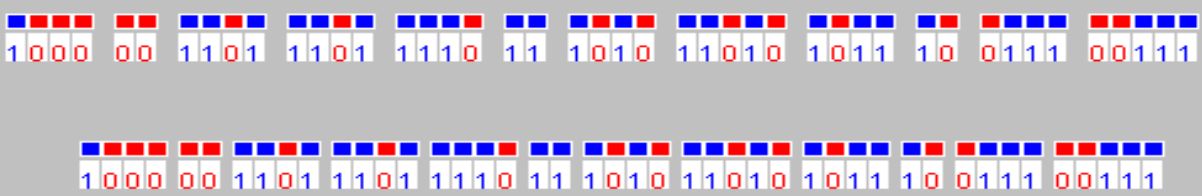

Fig 2 : Binary encoded chromosome

The selection operator was intended to improve the average quality of the population by giving the high- quality chromosomes a better chance to get copied into the next generation. Selection chromosomes are selected as parents to produce children and the chromosome with the best fitness values get selected to become the parent. The Tournament selection technique was used to pick a small subset of chromosomes (two or three) from the mating pool randomly.

Crossover exchanges information between two parent chromosomes in order to produce two new offsprings for the next population. A modified uniform crossover was presented, where one offspring was constructed by choosing every sub-chromosome with a probability $\mathrm{P}(=0.64)$ from either parent, as shown in Figure 3.

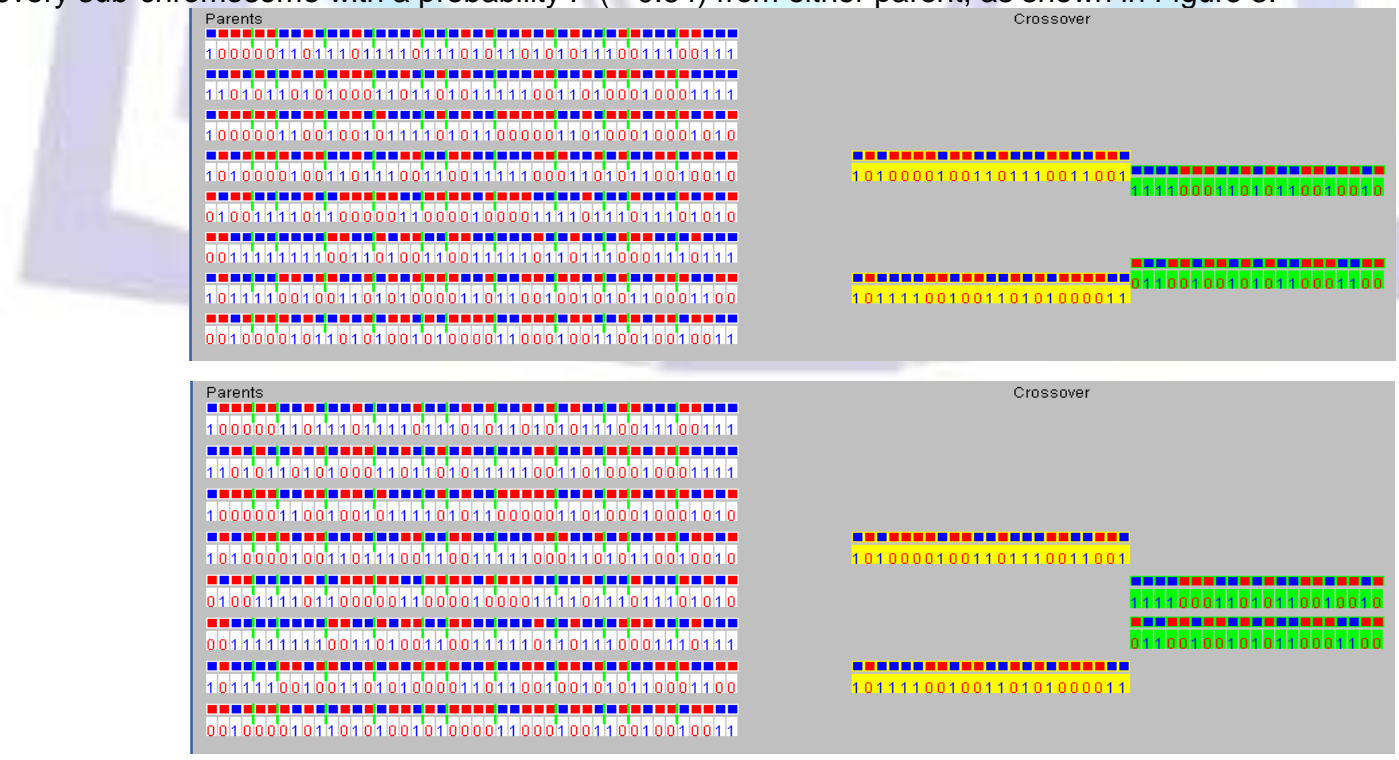

Fig 3: The first stage of crossover between two chromosomes.

The mutation operator is a random process where one genotype is replaced by another to generate a new chromosome. A single point mutation changes a 1 to a 0 , and vice versa. The mutation probability was 0.01 . 
Increasing the value of mutation probability increases the freedom to search outside the current feasible solution. The color of each mutated bit appeared as green impulse (Figure 4).

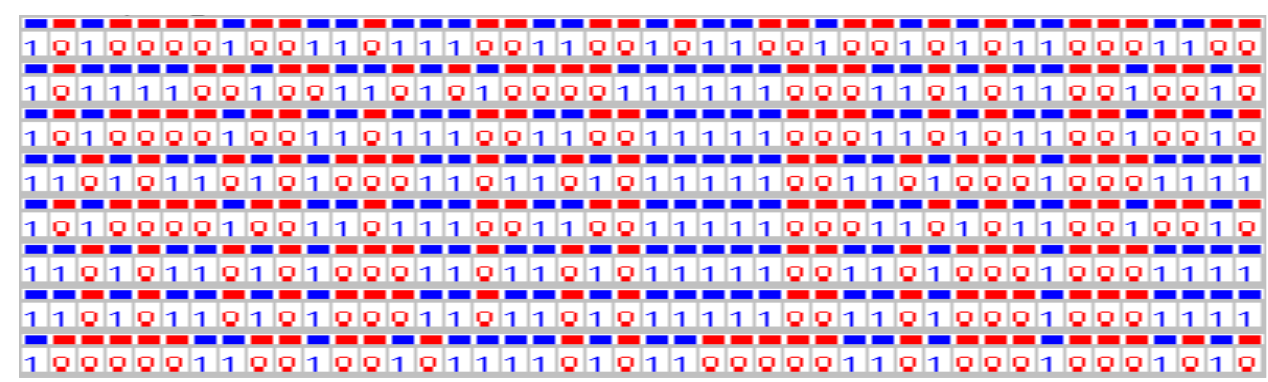

Fig 4: (a) Offspring before mutation

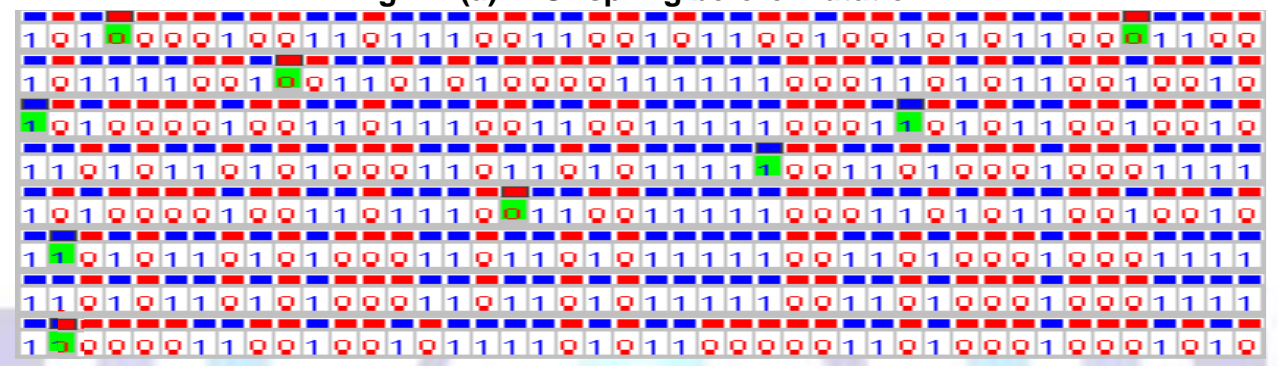

Fig 4: (b)

Offspring after mutation

\section{EXPERIMENTAL RESULTS}

Interactive algorithms (I \& II): Interactive methods may be characterized by the following steps: First, one or more non-dominated solutions are generated. Second, the decision maker is required for tradeoff information concerning these solutions, and the problem is modified based on the decision maker's responses. These two steps are then repeated iteratively until the decision maker is satisfied with the current solution.

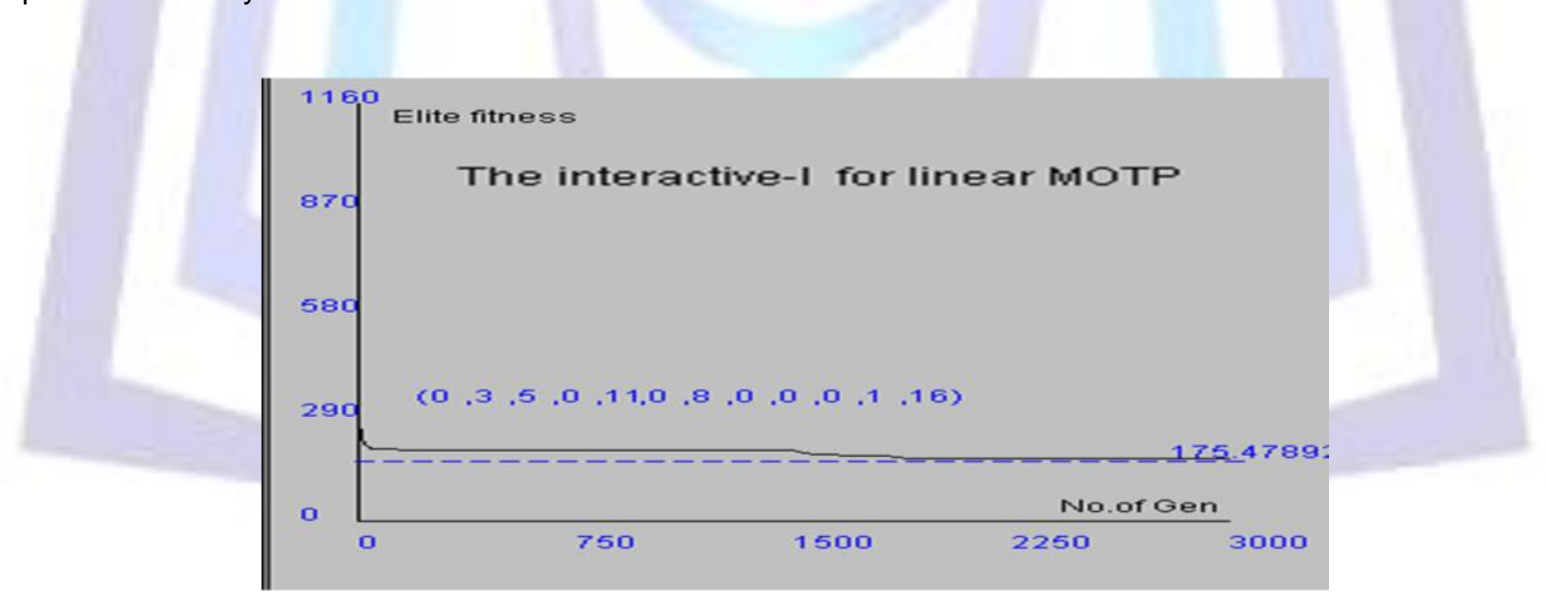

Fig 5: The chart represents the final solution using the Interactive Algorithms I

A fuzzy approach: The approach determined the optimal compromise solution of MOTP and studied the effect of using fuzzy programming on the MOTP model. The approach solved the single objective sub-problems and determined the upper and the lower bounds of each objective to construct the membership function of the MOTP problem. The result of the fuzzy programming approach showed the effect of using fuzzy programming on a special structure of the MOTP model. 


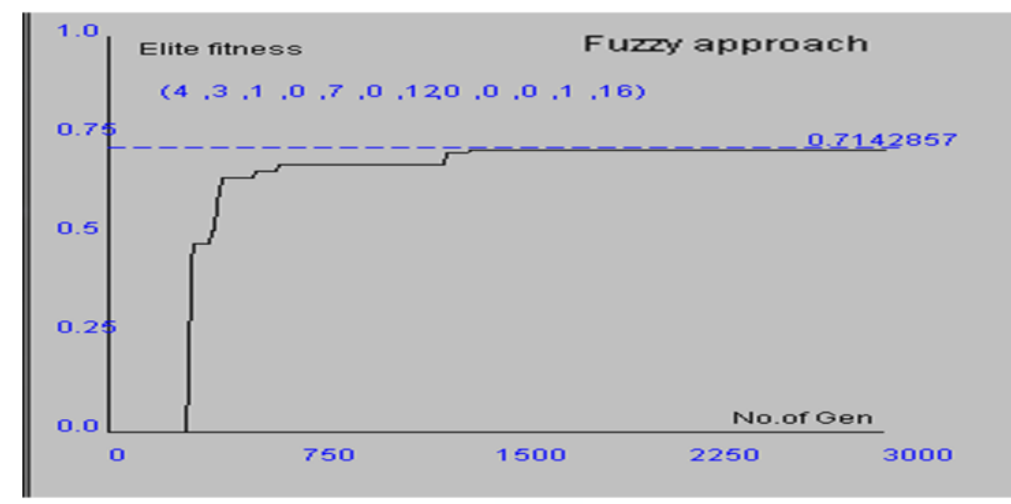

Fig 6: The chart represents the final solution using the Fuzzy Approach

1. Interactive fuzzy goal programming: This approach combined the goal programming, fuzzy programming and interactive programming in one methodology. Where, the basic idea of Goal Programming (GP) is a simple and easy to use. In GP, the decision maker should define the goal that he wants to accomplish. The GP model converts all the objective functions to constraints with the input of deviation variables from the goals. It can be expressed as the following:

$$
\min \left(\sum_{i=1}^{m}\left|F^{i}\left(X_{*}\right)-g_{i}\right|\right)
$$

The function $F^{k}\left(X_{*}\right)$ is the linear function of the $i^{t k}$ goal, and $g_{i}$ is the aspiration level of the $i^{t k}$ goal. The Interactive fuzzy goal programming approach controls the search direction via updating both upper bounds and aspiration level of each objective function. The solution results provide a preferred compromise solution which is more realistic from the decision maker perspective. The Interactive a fuzzy goal programming solution of MOTP can be summarized as; develop the MOTP as described in $P_{1}$, solve the first objective function as a single objective transportation problem, continue this process $K$ times for the $K$ objective functions. If all the solutions are the same, select one of them as an optimal compromise solution. Otherwise; determine the best lower bound and the worst upper bound to obtain a solution which is close to the best lower bound of each objective function. Develop problem $P_{1}$ and solve it as a GP problem.

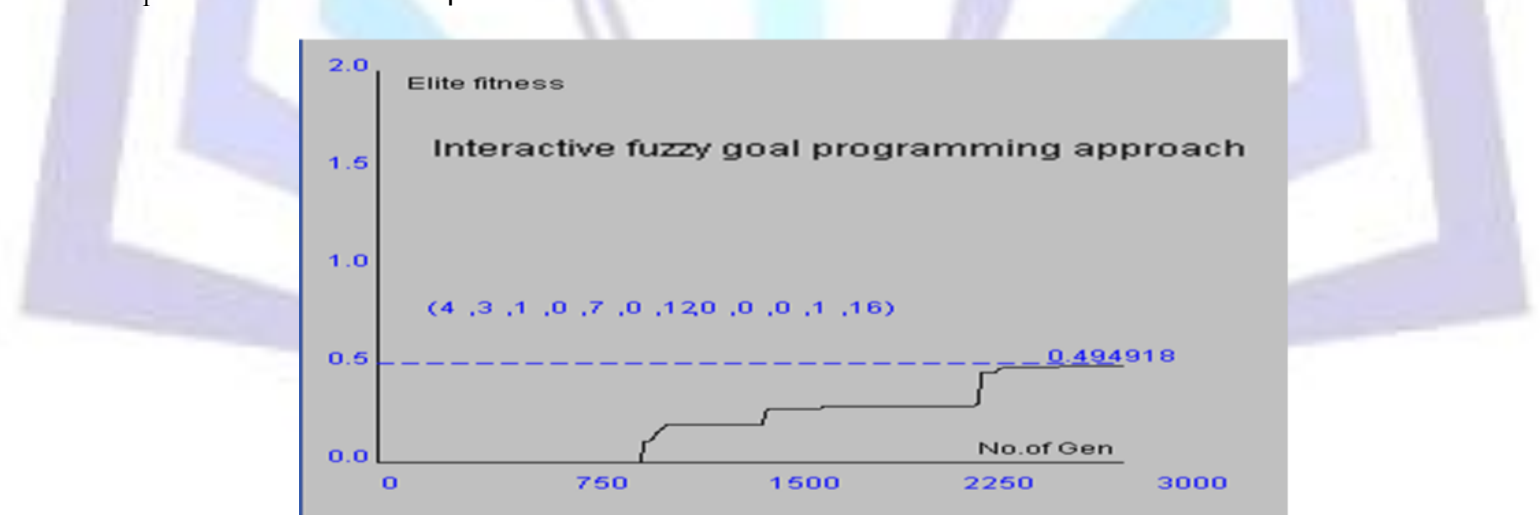

Fig 7: The chart represents the final solution using the Interactive Fuzzy Goal Programming Approach

2. Pareto-Based Approach: Pareto-based approach used non-dominated ranking and selection to move a population toward the Pareto front in MOTP. Non-dominated Sorting Genetic Algorithm was proposed and was based on several layers of classifications of the individuals. Before selection and mutation are performed, the population is ranked on the basis of non-domination. The Non-dominated Sorting Genetic Algorithm solved the service restoration problem in power distribution systems. This algorithm did not need weighting factors which were required for conversion of multi-objective function into an equivalent single objective function. 


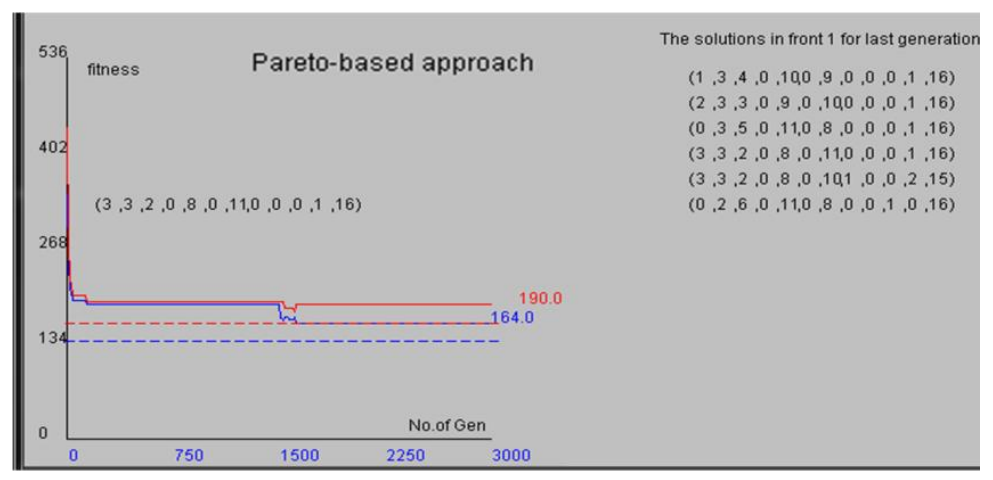

Fig 8: The chart represents the final solution using the Pareto-Based Approach

\section{STRATEGY OF REANALYSIS}

Thirty example of MOTP with three objective functions $F^{1}, F^{2}$ and $F^{3}$ were carried out using the four approachs; Paretobased approach, Interactive (I) approach, Fuzzy approach and Interactive Fuzzy goal approach. For each example, the program was run to obtain the compromise solution and calculate $F^{1}, F^{2}$ and $F^{3}$ and the distance function $D_{1}, D_{2}$ and $D_{\text {inf }}$.

Table 2 shows the the distance functions of the four approaches for solving MOTP. Regarding the distance function $D_{1}$, the Interactive (I) approach gave the least value $(0.229709)$ followed by the Pareto-based approach, the Fuzzy approach and the Interactive Fuzzy goal approach $(0.24328,0.274459$ and 0.246003 respectively).

However, the Pareto-based approach gave the least values of the distance function $D_{2}$ and $D_{\text {inf }}(0.148839$ and 0.109177 respectively) followed by the Interactive Fuzzy goal approach $(0.153074)$, Interactive (I) approach (0.153254) and Fuzzy approach $(0.163379)$ for the distance function $D_{2}$ and the Fuzzy approach $(0.116115)$, the Interactive Fuzzy goal approach (0.116964) and the Interactive (I) approach (0.122884) for $D_{\text {_inf }}$.

Table 2: The distance functions of the four approaches for solving the MOTP

\begin{tabular}{|c|c|c|c|}
\hline & $D_{1}$ & $D_{2}$ & $D_{- \text {inf }}$ \\
\hline Pareto-based approach & 0.24328 & 0.148839 & 0.109177 \\
\hline Interactive(I) approach & 0.229709 & 0.153254 & 0.122884 \\
\hline Fuzzy approach & 0.274459 & 0.163379 & 0.116115 \\
\hline Interactive Fuzzy goal approach & 0.246003 & 0.153074 & 0.116964 \\
\hline
\end{tabular}

The results suggested that the Pareto-based approach gave a preferred compromise solution which was better than the solution by the Interactive (I) approach, Interactive Fuzzy goal programming approach and Fuzzy approach for the distance functions $D_{2}$ and $D_{-}$inf. The good performance of the Pareto-based approach could be conveyed to the following reasons: Pareto-based approach aims to maintain diversity in the population instead of converging to a single solution and it uses non-domination to assess the fitness of individuals. This approach is dividing generation into a number of fronts. The best solution can be selected from the best front (the front that minimizes or maximizes the fittness function)[23].

The Interactive Fuzzy goal programming approach was relatively better than the Interactive (I) approach and Fuzzy approach. This could be explained by the lower value of the distance functions $D_{1}$ and $D_{2}$ obtained using the Interactive Fuzzy goal approach compared to the Interactive (I) approach. Also the value of the distance functions $D_{2}$ and $D_{-}$inf were lower for the Interactive Fuzzy goal approach than the Fuzzy approach. The good performance of the Interactive Fuzzy goal programming approach could be due to the fact that the Interactive Fuzzy goal programming approach is a compilation of goal programming approach and the fuzzy approach. Therefore, the Interactive Fuzzy goal programming approach is an improvement for Fuzzy approach. Moreover, the Interactive (I) approach was better than the Fuzzy approach based on the value of the distance functions $D_{1}$ and $D_{2}$.

\section{CONCLUSIONS}

This paper introduced a visual emplementation for the four approaches; Pareto-based approach, Interactive (I) approach, Fuzzy approach and Interactive Fuzzy goal approach for solving MOTP by object-oriented programming (Java). After running the java program for the four methods it could be concluded that the Pareto-based approach gave the best 
compromise solution followed by the Interactive Fuzzy goal approach, Interactive (I) approach and finally the Fuzzy approach.

\section{REFFERENCES}

[1] Abd El-Wahed, W.F.A multi-objective transportation problem under fuzziness, Fuzzy Sets and Systems, vol. 117, pp 27-33, 2001.

[2] Abd El-Wahed, W.F.and Lee, S. M, Interactive fuzzy goal programming for multi-objective transportation problems, Omega, vol34, pp 158 - 166, 2006.

[3] Charnsethikul Peerayuth and Sverasreni Saeree, (2007). The constrained bottleneck transportation problem. Journal of Mathematics and Statistics, 3:24-27.

[4] Charnes, Cooper, R Ferguson (1955) Optimal estimation of executive compensation by linear programming, Management Science, 1, 138-151.

[5] Deb, K., Pratap, A., Agarwal, S. and Meyarivan, T.:A fast and elitist multi-objective genetic algorithm: NSGA-II, IEEE Trans. Evol. Comput. vol. 6 (2), pp 182-197, 2002.

[6] F. Jimenez and J. L. Verdegay, Solving fuzzy solid transportation problems by an evolutionary algorithm based parametric approach, European Journal of Operational Research, 117 (1999), 485-510.

[7] J. Brito, J. A. Moreno and J. L. Verdegay, Transport route planning models based on fuzzy approach, Iranian Journal of Fuzzy Systems, 9(1) (2012), 141-158.

[8] J. L. Ringuest and D. B. Rinks, Interactive solutions for the linear multiobjective transportation problem, European J. Oper. Res., 32 (1987), 96-106.

[9] Khanna Saroj, Bakhshi H.C. and Puri M.C. (1983) Maximizing transportation flow under budgetary constraints, NZOR, 11:41-50.

[10] Kishore N. and Jayswal Anurag, (2001) Prioritized goal programming formulation of an unbalanced transportation problem with budgetary constraints, Industrial Engineering Journal,9:16-19.

[11] Kishore N. and Jayswal Anurag, (2002) Prioritized goal programming formulation of an unbalanced transportation problem with budgetary constraints: A fuzzy approach. Opsearch, 39:151-160.

[12] Kumar, Y., Das1, B. and Sharma, J.: Service restoration in distribution system using non-dominated sorting genetic algorithm, Electric Power Systems Research , vol 76, pp 768-777, 2006.

[13] Leberling $\mathrm{H}$. On finding compromise solutions in multi criteria problems using the fuzzy min-operator. Fuzzy Sets and Systems vol6, pp 18-105,1981.

[14] Lin Yi-Kuei and Yeh Cheng-Ta, (2011) Maximizing network reliability for stochastic transportation networks under a budget constraint by using a genetic algorithm. International Journal of Innovative Computing, Information and Control, 7:7033- 7050.

[15] Lohgaonkar M.H., Bajaj V.H., Jadhav V.A. and Patwari M.B. Fuzzy multi-objective multi-index transportation problem. Advances in Information Mining, ISSN: 0975-3265, Volume 2, Issue 1, 2010, pp-01-07

[16] Lushu, La.I. and Lai, K.K.: A fuzzy approach to the multi-objective transportation problem, Computers and Operational Research, vol27, pp 43-57, 2000.

[17] M. Zangiabadi and H. R. Maleki. Fuzzy goal programming technique to solve Multiobjective transportation problems with Some non-linear membership functions. Iranian Journal of Fuzzy Systems Vol. 10, No. 1, (2013) pp. 61-74

[18] Pandian P. and Natarajan G., (2010) A new method for finding an optimal solution for transportation problems. International J. of Math. Sci. and Engg. Appls., 4:59-65.

[19] Panos, M.P. and Donald, W.H.: Goal programming techniques for bank asset liability management, Applied Optimization, Vol.90, 2004 Kluwer Academic Publishers, Boston.

[20] Ringuest, J.L. and Rinks, D.B.: Interactive solutions for the linear multiobjective transportation problem, European Journal of Operational Research, vol32, pp 96-106,1987.

[21] S. M. Lee and L. J. Moore, Optimizing transportation problems with multiple objectives, AIEE Transactions, 5 (1973), 333-338.

[22] Senapati Samiran and Samanta Tapan Kumar, (2012) Optimal distribution of commodities under Budgetary restriction: a fuzzy approach. International Journal of Advanced Engineering Research and Studies, 1:208- 211.

[23] Steuer R. Multiple criteria optimization: theory, computation, and application. New York,Wiley; 1986.

[24] Tiwari R.N., Dharmar S. and Rao J. R. (1986) Priority structure in fuzzy Goal Programming. Fuzzy Sets and Systems, 19:251-259.

\section{Author's Biography}

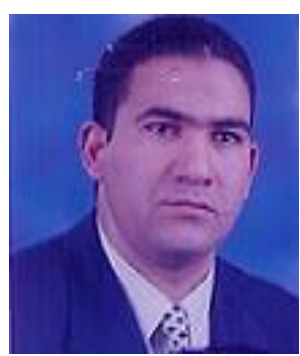

Prof. Dr. Mahmoud Moussa: I am a graduate of the Mathematics Department, the Faculty of Science, Benha University, Egypt in 1993 and graduated with grade Excellent with Honor. After being a demonstrator at Benha University, I was awarded my M. Sc. in 1999 in "Graph Algorithms", I was awarded my Ph.D. in 2005 in "Computer Science" at Karlsruhe University, German. I have moved to work in the Faculty of Computer Science and Information, Benha University in 2007. During my years of work at the Faculty of Computer Science and Information, Benha University, I have supervised my students in several. My interesting search areas are; Parallel Algorithms, Graph Algorithms, Graph Theory, Linear Programming, Labeling Graph, Applications on Matching Problems (Student Project Allocations Problem). and Wireless ad hoc networks and sensor networks 